\title{
Hypervelocity star candidates in Gaia DR1/TGAS
}

\section{T. Marchetti ${ }^{1}$, E. M. Rossi ${ }^{1}$, G. Kordopatis ${ }^{2}$, A. G. A. Brown ${ }^{1}$,} A. Rimoldi ${ }^{1}$, E. Starkenburg ${ }^{3}$, K. Youakim ${ }^{3}$ and R. Ashley ${ }^{4}$

${ }^{1}$ Leiden Observatory, Leiden University, PO Box 95132300 RA Leiden, the Netherlands email: marchetti@strw.leidenuniv.nl

${ }^{2}$ Université Côte d'Azur, Observatoire de la Côte d'Azur, CNRS, Laboratoire Lagrange, France

${ }^{3}$ Leibniz-Institut fur Astrophysik Potsdam (AIP), An der Sternwarte 16, 14482 Potsdam, Germany

${ }^{4}$ Department of Physics, University of Warwick, Gibbet Hill Road, Coventry, CV4 7AL, UK

\begin{abstract}
Hypervelocity stars (HVSs) are characterized by a total velocity in excess of the Galactic escape speed, and with trajectories consistent with coming from the Galactic Centre. We apply a novel data mining routine, an artificial neural network, to discover HVSs in the TGAS subset of the first data release of the Gaia satellite, using only the astrometry of the stars. We find 80 stars with a predicted probability $>90 \%$ of being HVSs, and we retrieved radial velocities for 47 of those. We discover 14 objects with a total velocity in the Galactic rest frame $>400 \mathrm{~km} \mathrm{~s}^{-1}$, and 5 of these have a probability $>50 \%$ of being unbound from the Milky Way. Tracing back orbits in different Galactic potentials, we discover 1 HVS candidate, 5 bound HVS candidates, and 5 runaway star candidates with remarkably high velocities, between 400 and $780 \mathrm{~km} \mathrm{~s}^{-1}$. We wait for future Gaia releases to confirm the goodness of our sample and to increase the number of HVS candidates.
\end{abstract}

Keywords. Astrometry, The Galaxy: kinematics and dynamics, The Galaxy: stellar content.

\section{Introduction}

Brown et al. (2005) discovered the first hypervelocity star (HVS): a star in the outer halo of the Milky Way with a heliocentric radial velocity of $\sim 850 \mathrm{~km} \mathrm{~s}^{-1}$, higher than the escape speed from the Galaxy. To explain such an extreme velocity, this star was suggested to be ejected according to the Hills mechanism, involving the interaction between a binary system and the massive black hole (MBH) in the Galactic Centre (GC) (Hills 1988). Observationally, what defines a HVS is that (i) its velocity is higher than the escape speed from the Galaxy at its position, and (ii) its trajectory is consistent with coming from the GC (Brown 2015). Following the first detection, a dedicated survey detected a total of 21 late B-type HVS candidates in the outer halo of the Milky Way (Brown et al. 2014).

HVSs have been proposed to study both the stellar population of the GC and the Galactic potential, since they can be used as dynamical tracers for the (dark) matter distribution (e.g. Gnedin et al. 2005, Yu \& Madau 2007). However results have been hampered by the quality and quantity of the current sample (Rossi et al. 2017).

The first release of the Gaia satellite (DR1, Gaia Collaboration et al. 2016a,b), and in particular the TGAS subset (Lindegren et al. 2016), gives us the first opportunity to increase the current sample of HVSs. The challenge is in the disparity between the expected number of HVSs and that of other bound stars in our Galaxy. For all the details on the search for HVSs in Gaia DR1, see Marchetti et al. (2017). To estimate how many 
HVSs we expect to find in the Gaia catalogue, we create mock populations using different assumptions for the unknown mass function, star formation rate, and binary population in the GC (Marchetti et al. in preparation). For the end of the mission catalogue, we anticipate from few hundreds to a thousand HVSs with relative error on total proper motion below $1 \%$, and thousands with relative error on total proper motion below $10 \%$. A few tens of objects will have relative error on parallax below 10\%. Around $100 \mathrm{HVSs}$ will be bright enough to have a radial velocity measurement. Repeating the same analysis considering the completeness limit of TGAS, we expect to find at maximum a few HVSs in this first release.

\section{Data Mining Routine and Application to Gaia DR1/TGAS}

In order not to bias our search towards particular spectral types, and to make as few assumption as possible on the stellar population in the GC, we decide to look for HVSs using only the information provided by TGAS: the 5 parameters astrometric solution. This consist of the projected position of the source on the sky, the parallax, and the two proper motions. We decide for a machine learning algorithm: an artificial neural network, trained on mock populations of HVSs and "normal" bound stars, the great majority of objects that Gaia will observe. We use the same mock populations mentioned in $\S 1$ for modelling HVSs, and we take normal stars from the Gaia Universe Model Snapshot (GUMS, Robin et al. 2012). This combined catalogue of the five parameters for the two populations is then used as a labelled training set for the neural network, which learns the general function mapping inputs (the five parameters) into the output (predicted probability of being a HVS).

The blind application of the neural network to the complete TGAS catalogue $(2057050$ sources) results in a total of 22263 stars with predicted probability $>50 \%$ of being HVSs, approximately $1 \%$ of the full catalogue. If we further require relative error on parallaxes to be lower than 1 , we are left with 8175 stars, $\sim 0.4 \%$ of the initial number of sources. We include uncertainties using Monte Carlo (MC) simulations: we draw 1000 realizations of the astrometry of each source, and we study the distribution of the output $D$ of the neural network. We define our best candidates as those with $\bar{D}-\sigma_{D}>0.9$, where $\bar{D}$ and $\sigma_{D}$ are, respectively, the mean and the standard deviation of the probability distribution of the parameter $D$. This cut results in a total of 80 HVS candidates, selected without any prior assumption on the stellar type or on the photometry.

\section{Results}

In order to confirm or reject the nature of our candidates as HVSs, we need a measure of their total velocity. We successfully applied for director's discretionary time at the Isaac Newton Telescope (INT) in La Palma, Canary Island, where, on the night of the 5th of October 2016, we followed up spectroscopically 22 candidates. Furthermore, we crossmatch our final sample with several spectroscopic surveys of the Milky Way. We are then able to recover a radial velocity measurement for 47 out of 80 stars. To derive distances from TGAS parallaxes, we implement a Bayesian approach similar to Astraatmadja \& Bailer-Jones (2016), but considering also covariances between astrometric parameters. In addition, we determine spectroscopic distances for 22 stars. In the following we will present results assuming both distance estimates.

In Figure 1 we plot the total velocity in the Galactic rest frame $v_{\mathrm{GC}}$ as a function of Galactocentric distance for the 47 candidates with a reliable radial velocity measurement. Error bars are dominated by distance uncertainties. The left (right) panel corresponds 


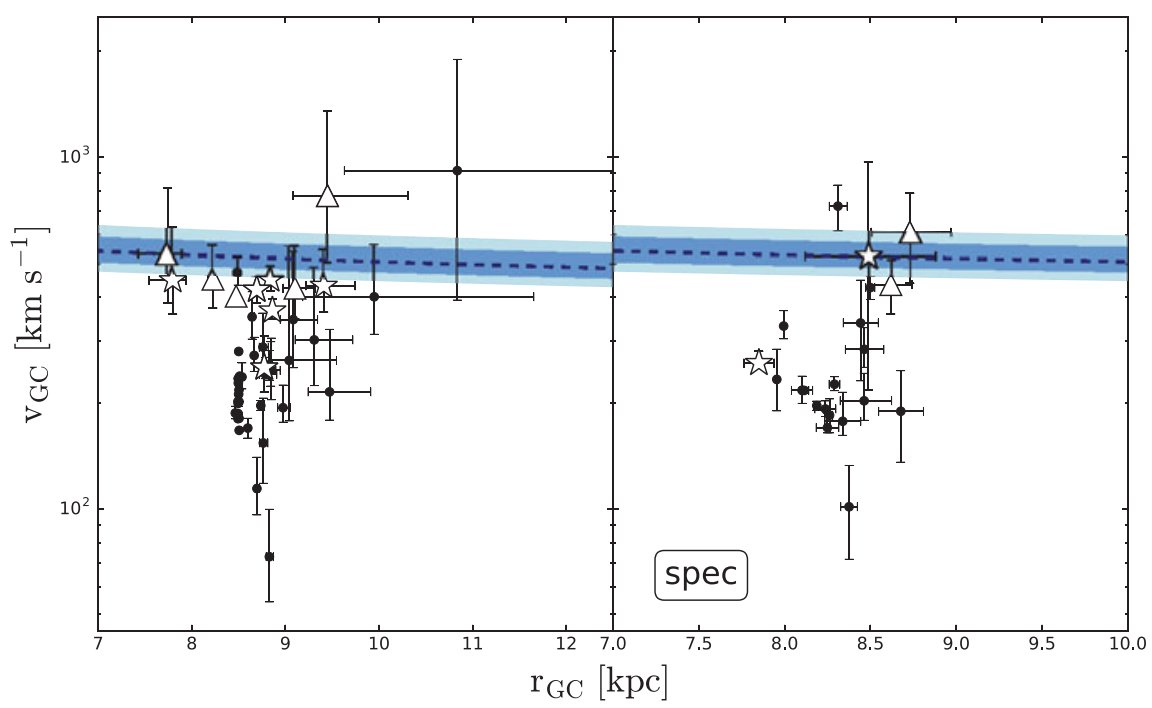

Figure 1. Total Galactic rest-frame velocity as a function of galactocentric distance for HVS candidates with a radial velocity information, using parallax-inferred distances (left panel) and, when available, spectroscopic distances (right panel). Stars (triangles) mark HVS/BHVS (runaway star) candidates, as in Table 1 . The dashed line corresponds to the median escape velocity from the Milky Way (Williams et al. 2017), with the 68\% (94\%) credible interval shown as a dark (light) band.

to velocities computed adopting parallax-inferred (spectroscopic) distances. We overplot with a dashed line the median escape speed from the Milky Way from Williams et al. (2017), with corresponding 68\% (94\%) credible intervals shown as a dark (light) region. This plot shows how our algorithm succeeded in finding high velocity stars: 45 stars have median $v_{\mathrm{GC}}>150 \mathrm{~km} \mathrm{~s}^{-1}$, and 14 of these have median $v_{\mathrm{GC}}>400 \mathrm{~km} \mathrm{~s}^{-1}$. A total of 11 (3) stars are consistent with being unbound from the Galaxy in the left (right) panel.

To assess whether our candidates come from the GC, we integrate their orbits back in time in different Galactic potentials using the python package galpy (Bovy 2015). We draw $10^{5}$ realizations of the initial condition of each star, and we check the consistency of the GC origin hypothesis for our candidates by recording the closest disc crossing (Galactic latitude $=0$ ) to the GC. We find 8 (3) stars to have orbits consistent with coming from the GC using parallax-inferred (spectroscopic) distances. We define the probability of each star of being unbound from the Milky Way, $P^{u}$, as the fraction of MC realizations resulting in a total velocity higher than the escape speed at that position.

We classify stars as HVSs if (i) their velocity is $>350 \mathrm{~km} \mathrm{~s}^{-1}$ using at least one distance estimate, and (ii) if they are consistent with coming from the GC within $2 \sigma$ when tracing back their obits. The Hills mechanism predicts as well a population of bound hypervelocity stars (BHVSs): stars with a velocity high enough to escape from the gravitational field of the $\mathrm{MBH}$, but not sufficient to be unbound from the Galaxy (e.g. Bromley et al. 2006). Stars are then sub-classified as HVSs (BHVSs) if $P^{u}>50 \%\left(P^{u}<50 \%\right)$. We classify as runaway stars those objects with a velocity $>350 \mathrm{~km} \mathrm{~s}^{-1}$ and with orbits not consistent with coming from the GC. The 11 candidates whose ejection location interpretation is independent from the adopted distance are presented in Table 1. According to the adopted classification, we discover 1 HVS candidate, 5 BHVS candidates, and 5 runaway star candidates. Interestingly, 2 runaway stars have probabilities $>50 \%$ of being unbound from our Galaxy. One possible acceleration mechanism for these stars involves an asymmetric supernova explosion in a binary system (e.g. Tauris 2015). 
Table 1. Derived kinematic properties for HVS, BHVS and runaway star candidates.

\begin{tabular}{|c|c|c|c|c|c|c|}
\hline Tycho 2 ID & $\begin{array}{c}d \\
(\mathrm{pc})\end{array}$ & $\begin{array}{c}d_{\mathrm{spec}} \\
(\mathrm{pc})\end{array}$ & $\begin{array}{c}v_{\mathrm{GC}} \\
\left(\mathrm{km} \mathrm{s}^{-1}\right)\end{array}$ & $\begin{array}{c}v_{\mathrm{GCspec}} \\
\left(\mathrm{km} \mathrm{s}^{-1}\right)\end{array}$ & $P^{\mathrm{u}}$ & $P_{\text {spec }}^{\mathrm{u}}$ \\
\hline \multicolumn{7}{|l|}{ HVS / BHVS candidates } \\
\hline $2298-66-1$ & $431_{-55}^{+78}$ & $754 \pm 569$ & $248_{-38}^{+58}$ & $519_{-307}^{+451}$ & $0.1 \%$ & $50.3 \%$ \\
\hline $8422-875-1^{1}$ & $1010_{-218}^{+400}$ & $208 \pm 124$ & $446_{-89}^{+186}$ & $259_{-7}^{+21}$ & $29.1 \%$ & $0.0 \%$ \\
\hline $2456-2178-1$ & $976_{-207}^{+358}$ & & $430_{-68}^{+117}$ & & $22.7 \%$ & \\
\hline $2348-333-1$ & $407_{-40}^{+51}$ & & $448_{-32}^{+44}$ & & $7.6 \%$ & \\
\hline $49-1326-1$ & $304_{-30}^{+38}$ & & $419_{-35}^{+38}$ & & $1.2 \%$ & \\
\hline $5890-971-1$ & $550_{-72}^{+93}$ & & $366_{-20}^{+29}$ & & $0.2 \%$ & \\
\hline \multicolumn{7}{|c|}{ Runaway star candidates } \\
\hline $7111-718-1$ & $1967_{-683}^{+1413}$ & $1552 \pm 430$ & $776_{-274}^{+576}$ & $611_{-172}^{+176}$ & $82.2 \%$ & $70.7 \%$ \\
\hline $8374-757-1$ & $832_{-179}^{+338}$ & & $532_{-147}^{+284}$ & & $50.4 \%$ & \\
\hline 1071-404-1 & $439_{-64}^{+91}$ & & $449_{-78}^{+113}$ & & $23.7 \%$ & \\
\hline $4515-1197-1$ & $881_{-175}^{+292}$ & $902 \pm 170$ & $423_{-76}^{+137}$ & $433_{-76}^{+78}$ & $23.5 \%$ & $15.6 \%$ \\
\hline $9404-1260-1$ & $67.0_{-0.9}^{+1.0}$ & & $402_{-4}^{+4}$ & & $0.0 \%$ & \\
\hline
\end{tabular}

1 The parallax-inferred distance $d$ is more likely to be correct: this object is a RR Lyrae star, and this estimate consistent with the value obtained using a PLZ relation.

Notes: The subscript "spec" refers to quantities computed using the spectroscopic distance. Results are quoted in terms of the median of the distribution with uncertainties derived from the 16th and 84 th percentiles.

With the advent of future Gaia releases we will be able to confirm the nature of our candidates, narrowing down their ejection location. Moreover, we will be able to use radial velocity as an extra feature to achieve a more precise classifier, dramatically increasing the number of expected HVSs.

This work has made use of data from the European Space Agency (ESA) mission Gaia (https://www.cosmos.esa.int/gaia), processed by the Gaia Data Processing and Analysis Consortium (DPAC, https://www.cosmos.esa.int/web/gaia/dpac/consortium). Funding for the DPAC has been provided by national institutions, in particular the institutions participating in the Gaia Multilateral Agreement.

\section{References}

Astraatmadja, T. L. \& Bailer-Jones, C. A. L. 2016, ApJ, 832, 137

Bovy, J. 2015, ApJS, 216, 29

Bromley, B. C., et al. 2006, ApJ, 653, 1194

Brown, W. R., Geller, M. J., Kenyon, S. J., \& Kurtz, M. J. 2005, ApJ, 622, L33

Brown, W. R., Geller, M. J., \& Kenyon, S. J. 2014, ApJ, 787, 89

Brown, W. R. 2015, ARA $\& A, 53,15$

Gaia Collaboration et al. 2016a, A\&A, 595, A1

Gaia Collaboration et al. 2016b, A\&A, 595, A2

Gnedin, O. Y., Gould, A., Miralda-Escudé, J., \& Zentner, A. R. 2005, ApJ, 634, 344

Hills, J. G. 1988, Nature, 331, 687

Lindegren, L., et al. 2016, A\&A, 595, A4

Marchetti, T. et al. 2017, MNRAS, stx1304

Robin, A. C., et al. 2012, A\&\&A, 543, A100

Rossi, E. M., Marchetti, T., Cacciato, M., Kuiack, M., \& Sari, R. 2017, MNRAS, 467, 1844

Tauris, T. M. 2015, MNRAS, 448, L6

Williams, A. A., Belokurov, V., Casey, A. R., \& Evans, N. W. 2017, MNRAS, 468, 2359

Yu, Q. \& Madau, P. 2007, MNRAS, 379, 1293 